\title{
ON GENERALIZATION OF MOSER'S THEOREM IN THE CRITICAL CASE
}

\section{ROBERT ČERNÝ AND SILVIE MAŠKOVÁ}

Abstract. Let $\Omega$ be an open bounded set in $\mathbb{R}^{n}, n \geqslant 2$. In paper [13] Moser proved that for every $K \geqslant K_{0}=n^{-\frac{n-1}{n}} \omega_{n-1}^{-\frac{1}{n}}$ we have

$$
\sup \left\{\int_{\Omega} \exp \left(\left(\frac{f(x)}{K}\right)^{\frac{n}{n-1}}\right): f \in W_{0}^{1, n}(\Omega),\|\nabla f\|_{L^{n}} \leqslant 1\right\}<\infty,
$$

but for $K<K_{0}$ the supremum is not finite.

In this paper we study the critical case $K=K_{0}$ for arbitrary Orlicz-Sobolev spaces with Young functions that behave like $t^{n}$ close to $\infty$. We show that for functions like $t^{n}\left(1-\log ^{-a} t\right)$ the supremum is finite for $a>1$ but infinite for $0<a<1$.

Mathematics subject classification (2010): 46E35,46E30.

Keywords and phrases: Orlicz spaces; Orlicz-Sobolev spaces; embedding theorems; sharp constants.

\section{REFERENCES}

[1] D. R. Adams, L. I. Hedberg, Function spaces and Potential theory, Springer, 1996.

[2] A. Cianchi, A sharp embedding theorem for Orlicz-Sobolev spaces, Indiana Univ. Math. J., 45, (1996), 39-65.

[3] D.E. Edmunds, P. GuRKa, B. Opic, Double exponential integrability of convolution operators in generalized Lorentz-Zygmund spaces, Indiana Univ. Math. J., 44 (1995), 19-43.

[4] D.E. Edmunds, P. Gurka, B. OpIC, Double exponential integrability, Bessel potentials and embedding theorems, Studia Math., 115 (1995), 151-181.

[5] D.E. Edmunds, P. GuRKA, B. OpIC, Sharpness of embeddings in logarithmic Bessel-potential spaces, Proc. Roy. Soc. Edinburgh, 126A (1996), 995-1009.

[6] D.E. Edmunds, P. GuRKA, B. OpIC, On embeddings of logarithmic Bessel potential spaces, J. Funct. Anal., 146 (1997), 116-150.

[7] D.E. Edmunds, P. GuRKA, B. OPIC, Norms of embeddings in logarithmic Bessel-potential spaces, Proc. Amer. Math. Soc., 126 (1998), 2417-2425.

[8] D.E. Edmunds, M. Krbec, Two limiting cases of Sobolev imbeddings, Houston J. Math., 21 (1995), 119-128.

[9] N. Fusco, P. L. Lions, C. Sbordone, Sobolev imbedding theorems in borderline cases, Proc. Amer. Math. Soc., 124 (1996), 561-565.

[10] A. M. Garsia, Letter to J. Moser, March 29, 1972.

[11] L. I. Hedberg, On certain convolution inequalities, Proc. Amer. Math. Soc., 36 (1972), $505-512$.

[12] S. HENCL, A sharp form of an embedding into exponential and double exponential spaces, J. Funct. Anal, 204 (2003), 196-227.

[13] J. Moser, A sharp form of an inequality by N. Trudinger, Indiana Univ. Math. J., 20 (1971), 10771092.

[14] B. OpIC, L. PICK, On generalized Lorentz-Zygmund spaces, Math. Inequal. Appl., 2 (1999), $391-467$.

[15] M. M. RaO, Z. D. REN, Theory of Orlicz spaces, Pure and applied mathematics, 1991.

[16] R. S. Strichartz, A note on Trudinger's extension of Sobolev's inequality, Indiana Univ. Math. J., 21 (1972), 841-842. 
[17] G. Talenti, Inequalities in rearrangement invariant function spaces, Prometheus Publ. House Prague, Nonlinear Analysis, Function Spaces and Applications, 5 (1994), 177-230.

[18] N. S. Trudinger, On imbeddings into Orlicz spaces and some applications, J. Math. Mech., 17 (1967), 473-484.

[19] V. I. YUDOVIČ, Some estimates connected with integral operators and with solutions of elliptic equations, Soviet Math. Doklady, 2 (1961), 746-749. 